\title{
TEMPORAL 24-HOUR ASSESSMENT OF
}

\section{RADIO FREQUENCY EXPOSURE IN}

\section{SCHOOLS AND HOMES}

Leen Verloock*, Wout Joseph*, Francis Goeminne*, Luc Martens*, Mart Verlaek**, and Kim Constandt**

(email:wout.joseph@intec.UGent.be, fax:+32 933 14899)

*Department of Information Technology, Ghent University / iMinds Gaston Crommenlaan 8, box 201, B-9050 Ghent, Belgium

** Department of Environment, Nature and Energy (LNE), Flemish government, Koning Albert IIlaan 20, box 8, B-1000 Brussels, Belgium

Abbreviated title: 24-hour exposure in schools and homes 
Abstract- Temporal radio frequency (RF) exposure from present and emerging technologies in sensitive microenvironments such as schools and homes is important for evaluations of compliance to international limits. For the first time, temporal 24-hour measurements of all present RF signals, including LTE (Long term Evolution), are performed with accurate spectral narrowband equipment in these environments where children are present. The largest maximal variations are obtained for the cordless telephony (DECT) signals (10.6 dB) and for the WiFi $2.4 \mathrm{GHz}$ signals $(12.7 \mathrm{~dB})$, while variations of broadcasting signals and telecommunication signals were much lower namely, $2.9 \mathrm{~dB}$ and $3.3 \mathrm{~dB}$, respectively. Thus, indoor sources exhibit the largest variations indoor and are the most critical for practical exposure assessment and comparison with existing guidelines. It is recommended to perform exposure measurements during school days as highest field values were measured then. All field values measured over 24 hours satisfied the ICNIRP reference levels.

Key Words- temporal exposure, 24 hours, school, home, RF exposure of general public, electromagnetic measurement.

\section{Highlights}

- Temporal exposures during 24 hours are assessed in schools and homes

- All present RF signals are included in the analysis including LTE

- Signals from indoor sources DECT and WiFi have largest variations

- We determine for which periods of the day higher variations are possible.

- $\quad$ The WiFi duty cycle (actual usage) is measured and characterized during 24 hours. 


\section{INTRODUCTION}

Taking temporal variations of exposure to wireless networks into account when assessing exposure for compliance with existing exposure limits [1] is a must. Because of the rapid evolution of wireless technologies, it is important to permanently monitor exposure from radiofrequency electromagnetic fields (RF-EMF), as stated in the World Health Organization's (WHO) research agenda [2]. Up to now, data and assessment of temporal variations of RF-EMF exposure in sensitive places such as schools and homes are lacking. Measurements during time (days, weeks) are time-consuming, expensive, and difficult to execute in practice. Therefore compliance is often only evaluated by performing short-time spatial measurements $[3,4,5,6,7]$.

Only limited data about temporal variations are available [8, 9, 10, 11, 12, 13, 15]. Existing literature and procedures are discussed in detail in [8] and [10] and a CENELEC standard has been proposed for the in-situ measurement of electromagnetic-field strength [16]. Manassas et al. [9] investigated diurnal variations of fields due to broadcasting and mobile telecommunication and provided median variations. In [10], Erlang data (representing average mobile phone traffic intensity during a period of time) is related to RF exposure using temporal measurements during a week. [11] compared real exposure with the maximal estimated exposure to characterize the ratio between daily and maximum theoretical exposure values, while [12] compared various realistic extrapolation methods in two countries. The authors of [13] also stated that short-term exposure assessment is not reliable for evaluation of long-term exposure. [14] reported a variability of power density of the GSM900 downlink band of $\pm 5.2 \mathrm{~dB}$ throughout the day as a result of traffic variation. The measurements of [14] were executed indoor at a single location. RF-EMF exposure assessment studies using exposimeters have also been reported, investigating spatial and temporal field aspects [17, 18, 19, 20] but these assessments are difficult for accurate evaluation of temporal exposures due to limitations of the exposimeters (no settings per signal possible) and the use of exposimeters on the body can influence the results enormously. Finally, [15] proposed a 
combination of temporal exposimeter measurements (standing alone) and spatial spectral narrowband measurements to assess exposure in indoor environments.

Currently, temporal RF-EMF exposure assessment in "sensitive” environments such as schools and homes, where children reside, is missing. The objective of this paper is to assess and characterize temporal exposures with accurate narrowband equipment (spectrum analyzers and measurement probes) during 24 hours in schools and homes where children are present. All present RF signals, including LTE (Long Term Evolution, recently being rolled-out in Belgium since the end of 2012) are considered. Moreover, we determine for which periods of the day (morning, school day, evening, night) higher variations of the exposure are possible in the different microenvironments. Finally, for WiFi, the duty cycle, which depends upon the type of wireless traffic and actual usage, is measured and characterized during 24 hours in these environments. Up to now, the assessment of the WiFi duty cycle was only performed at specific moments or for specific applications for WiFi. Authorities can use the recommendations and results of this paper to determine the time and moment of day when evaluating compliance to their guidelines. Moreover, the results are of importance for the WHO to build up knowledge about the temporal variations of emerging technologies.

This work was in authorization of the Flemish government. The Flemish government, Department of Environment, Nature and Energy (LNE) commissioned this study and no separate ethical approval was needed as the measurements did not occur with children but in their living environment. Approval from schools and Flemish government was obtained. 


\section{MATERIALS AND METHOD}

\subsection{Selection of microenvironments}

At 10 microenvironments, 5 schools and 5 homes, 24-hour temporal measurements were performed. All temporal measurements occurred indoor. The microenvironments are located in urban environments. The schools were selected based on the presence of internal RF sources and the use of wireless local area network (WLAN) devices. In every school, WiFi is used as WLAN technology and in a school tablets were used for educational purposes. Also five homes where children reside and WiFi was present were investigated. The homes are regular houses; no apartment buildings or flats were considered. The measurements were performed in the period of October 2012 - April 2013.

\subsection{Measurement equipment}

Temporal exposure variations were assessed using frequency-selective narrowband measurements. The setup consisted of tri-axial Rhode and Schwarz R\&S TS-EMF isotropic antennas (dynamic range of $1 \mathrm{mV} / \mathrm{m}-100 \mathrm{~V} / \mathrm{m}$ for the frequency range of $80 \mathrm{MHz}-3 \mathrm{GHz}$, and $2.5 \mathrm{mV} / \mathrm{m}-200 \mathrm{~V} / \mathrm{m}$ for a frequency range of $2 \mathrm{GHz}-6 \mathrm{GHz}$ ) in combination with a spectrum analyzer (SA) of type R\&S FSL6 (frequency range of $9 \mathrm{kHz}-6 \mathrm{GHz}$ ) (R\&S, Munich, Germany). The measurement uncertainty was $\pm 3 \mathrm{~dB}$ for the considered setup [6], [16], [21]. This uncertainty represents the expanded uncertainty evaluated using a confidence interval of 95\%.

The location of the maximal total electric-field value at the site under consideration is identified through sweeping the area with the broadband probe [16]. A broadband probe of type Narda NBM-550 (measurement equipment) equipped with EF0391 (measurement probe with a dynamic range of $0.2-320 \mathrm{~V} / \mathrm{m}$ and a frequency range of $100 \mathrm{kHz}$ to $3 \mathrm{GHz}$ ) or EF0691 (measurement probe with a dynamic range of $0.35-650 \mathrm{~V} / \mathrm{m}$ and a frequency range of $100 \mathrm{kHz}$ to $6 \mathrm{GHz}$ ) was used for this purpose. 


\subsection{Measurement procedure to characterize the temporal variations}

Firstly, at each school and home, 24-hour temporal measurements (during working days) were performed at a specific location. The location for this assessment was selected as follows. With a broadband probe the locations of maximal fields were searched for. At the location of the maximal total field value, the setup for the temporal assessment was positioned if practically possible. One has often to take into account that children and adults are not allowed to touch the equipment and be careful for vandalism or theft. If it was not possible to place the equipment at the maximum field location, the nearest "safe" location was chosen. We are here mainly interested in the temporal variations of the different present signals.

Secondly, with a spectrum analyser and tri-axial antenna, a spectral overview measurement in the frequency range of $80 \mathrm{MHz}$ up to $3 \mathrm{GHz}$ was performed to identify present RF signals.

Thirdly, narrowband measurements of the momentary field values of the significantly present signals (FM, T-DAB, DVB-T (digital TV), GSM900, GSM1800, UMTS/HSPA, LTE, WiMAX, DECT, WiFi,....) were performed during 24 hours [8], [10]. All the abbreviations of these RF signals are explained below Table 1 . The optimal settings of the SA for various RF signals were selected from [6]. This sequence of measurements was repeated an entire week day (24 hours). The cycle time was about 3 minutes (setting of configuration of frequency band to be measured, sweeping of the SA, reading and transferring of the measurement traces, configuration of next frequency band, etc.). This duration also depends upon the number of frequency bands. If WiFi was measured the duration was prolonged with the duration of a max-hold measurement (about 3 minutes) and the duration of a 1-minute measurement per channel to assess the duty cycle [5]. Performing these measurements during 24 hours resulted for each significant RF signal in a total of 440 samples per measurement site and a total of about 71,000 samples for all signals and microenvironments together. 


\subsection{Parameters to characterize the temporal variations}

To compare the temporal variations of the RF signals, we define the dynamic range $D R$ and the parameters $D R_{E, \max }$ and $D R_{E, 99 .} D R_{E, \max }$ is defined as the ratio of the maximal momentary electric-field value to the minimum electric-field value over the considered time interval (here 24 hours).

$$
D R_{E, \max }=20 * \log \left(\frac{\max \left(E_{\text {mom }}\right)_{24 \text { hours }}}{\min \left(E_{\text {mom }}\right)_{24 \text { hours }}}\right) \quad(d B)
$$

To eliminate the influence of outliers we define also $D R_{E, 99}$ as the ratio of the $99^{\text {th }}$ percentile (p99) of the momentary electric field value to the 1-percentile $\left(p_{1}\right)$ electric field value over the considered time interval of 24 hours.

$$
D R_{E, 99}=20 * \log \left(\frac{p_{99}\left(E_{\text {mom }}\right)_{24 \text { hours }}}{p_{1}\left(E_{\text {mom }}\right)_{24 \text { hours }}}\right)
$$

The higher the $D R$ values, the larger the variation of the signal during time. Further, we consider also the standard deviation $\sigma_{E}$, which is also a measure of the variation of the RF signals.

If the WiFi signal level is sufficiently high, then also the duty cycle $(D C)$ of the dominating channels is measured. The WiFi duty cycle DC is defined in [5] as the ratio of active duration $t_{\text {active }}(\mathrm{s})$ to total duration $t_{\text {tot }}(\mathrm{s})$ of the WLAN signal. We use here the method described in [5] to assess the duty cycles. Analogously to the electric-field levels, dynamic ranges can then be defined namely $D R_{D C \text {, max }}$ and $D R_{D C, 99}$. Also the standard deviation $\sigma_{D C}$ is determined. $D R_{D C, \max }$ and $D R_{D C, 99}$ will also be expressed in $\mathrm{dB}$ for comparison purposes.

We distinguish external sources (broadcast and telecommunication signals) and internal sources. Internal sources are transmitters that are located indoors and are placed by schools, private persons, or authorities and can be controlled (e.g., WiFi access points), while external sources are all other sources from broadcasting and telecommunication and cannot be controlled by the private persons or companies themselves (e.g., FM, PMR, TETRA, T-DAB, DVB-T, GSM, 
UMTS/HSPA, LTE, etc., see also footnote of Table 1). Telecommunication signals include here GSM900, GSM1800, UMTS/HSPA, TETRA, and LTE. Broadcasting signals include FM, T-DAB, DVB-T. DECT and WiFi sources are considered separately and are the internal signals. This categorization enables us to make better conclusions about the temporal variations and was also partly used in [9].

Finally, to enable comparison with exposure limits, the exposure ratio $(E R)$ of a signal ( $E R$ in \%) is defined as the ratio of the maximal measured electric-field value of the considered signal over the various positions and the corresponding ICNIRP reference level for general public. Exposure ratios smaller than $100 \%$, indicate compliance with the ICNIRP guidelines [1].

\section{RESULTS AND DISCUSSION}

\subsection{Temporal variations in the schools and homes}

As an example, Figure 1 shows the variation of the momentary field values $E_{\text {mom }}$ of relevant external RF signals over 24 hours (including the present LTE signals) in schools and homes. Fig. 2 compares the temporal variations of the internal DECT and WiFi signals.

All signals in Figs. 1 and 2 satisfy the ICNRIP reference levels for the entire period of 24 hours. The LTE signal in Fig. 1h does not vary much during time as only a limited number of users are using this new technology. Also broadcasting signals as FM, DVB-T, and T-DAB (Fig. 1a, b, and c) do not vary much here as there are no traffic variations compared to telecommunication signals. The broadcasting signals are constant during time and variations occur due to environmental changes (movement of people, etc.). Clearly, WiFi values in Fig. 2 are the highest during the school day because children and teachers are using wireless applications then. For this example, largest variations occur for TETRA (Fig. 1d), GSM900 (Fig. 1e), GSM1800 (Fig. 1f), and DECT signals (maximal standard deviation up to $1.5 \mathrm{~dB}$ and $D R_{E, 99}$ value up to $7.2 \mathrm{~dB}$ for the example). For telecommunication signals GSM and UMTS/HSPA both the environmental changes and the usage traffic cause variations [8], [10]. 
Figure 3 shows the variation of the WiFi duty cycle in channel 11 as a function of time. The largest variation of the duty cycle occurs during the school day (see also Fig. 2). Variations in morning and night are similar and the smallest. The peaks at 8:00 and around 11:00-12:00 indicate the start-up and buffering of applications such as video streaming over WiFi resulting in a period with a high duty cycle followed by lower duty cycles as observed in [24].

Table 1 summarizes the average $D R_{E \text {,max }}$ value, $D R_{E, 99}$ value, and the standard deviation $\sigma_{E}$ of the momentary measured field values $E_{\text {mom }}$ for the different categories of RF signals in schools and homes. Also the maximal measured electric field strength $E_{\max }$ per signal type over 24 hours and the exposure ratio ER are provided. Table 1 shows that the largest maximal variations (averaged over all locations) are obtained for the DECT signals (10.6 dB) and for the WiFi $2.4 \mathrm{GHz}$ signals (12.7 dB). Maximal variations of 2.9 and $3.3 \mathrm{~dB}$ are obtained for broadcasting and telecommunication signals, respectively. Thus, internal signals have the largest variations indoor (all measurements occurred indoor) and are the most critical ones for spatial assessment and comparison with exposure guidelines. Averaged over all locations we obtain as dynamic ranges $\left(D R_{E, 99}\right) 2.3 \mathrm{~dB}, 2.6 \mathrm{~dB}, 8.7 \mathrm{~dB}$ and $8.5 \mathrm{~dB}$ for broadcasting, telecommunication, DECT, and Wifi, respectively. For all environments, the variations of broadcasting signals and telecommunication signals are similar and much lower than those of DECT and WiFi. Finally, Table 1 shows that for the considered schools and homes (at the location of the temporal assessment), the field values measured over 24 hours satisfy the ICNIRP reference levels. Clearly, highest fields are measured for DECT $(3.1 \mathrm{~V} / \mathrm{m}$ in schools), followed by WiFi $(1.7 \mathrm{~V} / \mathrm{m})$ and telecommunication signals $(1.1 \mathrm{~V} / \mathrm{m})$. The highest value of the exposure ratio is $5.2 \%$ (DECT). Although our study focused on the temporal variations, we found that field levels in homes were generally the lowest as reported in [17], [18]. In schools, more people are simultaneously present and thus use of more sources occurs. 


\subsection{Temporal variations on different moments of the day}

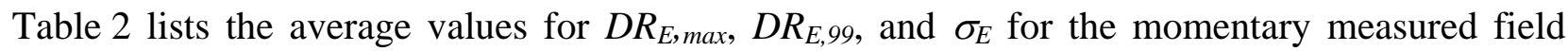
levels $E_{\text {mom }}$ for DECT, WiFi $2.4 \mathrm{GHz}$, and the external sources measured at different time instances. As variations of broadcasting and telecommunication signals are much lower than those of DECT and WiFi, we group them in external sources. We distinguish morning (6am to 8am), school day (8am to $4 \mathrm{pm}$ ), evening (4pm to 12pm), night (12pm to 6am). Table 2 shows that the largest variations occur during the school day for DECT and the external signals. In the evening the largest variations are measured for WiFi. As highest field values are measured during the school day, we recommend to perform exposure measurements during school days. At night and in the morning the variations of all signals are the lowest and very similar e.g., maximal 6.4 and 6.3 $\mathrm{dB}$ for DECT and 5.1 and $5.3 \mathrm{~dB}$ at night and in the morning, respectively and $2.1 \mathrm{~dB}$ for external signals (Table 2). This indicates that night and morning periods could be merged to a single period (for all considered signals) where only low usage traffic is present, which is logical as most people are sleeping or less active at these periods of the day. This conclusion agrees with the results of [11], [12] (3 periods, see further).

\subsection{Temporal variations of the WiFi duty cycle}

Table 3 lists the average $D R_{D C \text {,max }}, D R_{D C, 99}$, and $\sigma_{D C}$ for the duty cycle from the WiFi $2.4 \mathrm{GHz}$ signal in schools and homes. Largest variations are obtained for schools: up to $30.6 \mathrm{~dB}$ compared to maximally $6.4 \mathrm{~dB}$ in homes. Also the standard deviation in schools is much higher (about $3 \mathrm{~dB}$ compared to $0.9 \mathrm{~dB}$ ). Table 3 lists also again these values for the different time instances (morning, school day, evening, night). Highest variations for the duty cycle are again obtained during the school day $(12.7 \mathrm{~dB})$, variations are lower in the evening $(5 \mathrm{~dB})$ and the lowest at night and in the morning (about $2.0 \mathrm{~dB}$ ). Again the variations of the duty cycle during night and morning are almost the same (2.0 versus $1.9 \mathrm{~dB}$ for $D R_{D C \text {,max }}$ in Table 3). 


\subsection{Comparison of temporal variations with related research}

Up to now the focus of the research about temporal exposure assessment was on the telecommunication signals GSM900 and UMTS. [10], [13], [14] focused on GSM900, while [11], [12] also investigated GSM900, GSM1800, UMTS/HSPA. [8], [9] considered also broadcasting signals next to these telecommunication signals. Temporal variations of other signals were almost never investigated: [5] investigates only variations of the WiFi duty cycle over short periods according to ICNIRP [1] but not over 24 hours. The considered environments were mainly urban or rural and homes and schools were not considered up to now.

Variations of $\pm 4.5 \mathrm{~dB}$ and $\pm 5.2 \mathrm{~dB}$ for GSM900 as a result of traffic variations were obtained in [11] and [14], respectively. In [14], only one single measurement location was considered. [8], [10] obtained median variations over 7 days of $1.7 \mathrm{~dB}$ for FM and of about $7.5 \mathrm{~dB}$ for GSM900, GSM1800, and UMTS at outdoor locations. Median diurnal variations of 20.2\% (1.7 dB) and 33.8\% (3.6 dB) for the broadcasting and mobile telecommunication emissions, respectively, were reported in [9]. Temporal variations (assessed using exposimeters) of indoor exposure in Belgium and Greece were mainly due to variations of mobile telecommunication signals according to [15]. The exposure was higher during daytime than at night due to traffic on the networks. Highest variations occurred in Belgian crèches (39.3\% or $3.3 \mathrm{~dB}$ ) and Greek homes (58.2\% or $7.6 \mathrm{~dB})$. However, limitations in the study of [15] were the occurrence of incorrect field values due to changing the batteries of the exposimeters and the limited number of different indoor microenvironments. Moreover the exposimeters are not as sensitive as spectral equipment. Here, maximal variations of $3.3 \mathrm{~dB}$ (indoor) over 24 hours were obtained for telecommunication, which is similar to [11], [14], and [15]. Telecommunication includes here also the LTE signal, which is a new signal and varies less because there are currently only a limited number of users. Telecommunication signals (GSM and UMTS) vary more than broadcasting. 
Averaged over all locations we obtained as dynamic ranges $\left(\mathrm{DR}_{\mathrm{E}, 99}\right.$ : excluding maximum and minimum values to avoid outliers due to measurement errors) $2.3 \mathrm{~dB}, 2.6 \mathrm{~dB}, 8.7$ and $8.5 \mathrm{~dB}$ for broadcasting, telecommunication, DECT, and WiFi, respectively. Thus, the variations of DECT and WiFi (indoor) were much higher than these of the other signals, but this was not considered before in [8]- [14]. Our variations were thus slightly smaller than or similar to those reported above because we consider much more sites, different environments than in [8]-[14], and also emerging signals such as LTE, which vary less. Also the metric $\mathrm{DR}_{\mathrm{E}, 99}$ differs from the one used in [8]-[14], but it accounts for outliers.

For the temporal variations, 3 time periods were distinguished in [11], namely, night (no or little traffic), rush hour (maximum traffic), and working hours (normal use). These periods were modeled as Gaussian distributions resulting in a three Gaussians model. The 3-Gaussian method was used to estimate the maximum exposure in two countries in [12] (Belgium and France) and was compared with other temporal estimation methods such as those from [8], [10]. In [13], [14], 4 time intervals were proposed based on measurements at one location, depending on the traffic load: night hours, early-morning hours, work hours, and rest hours. Because of the school and home environment and the presence of children we distinguished here morning, school day, evening, and night (see Section 1.6). Our results indicated that (Table 2) night and morning (for the considered schools and homes) could be merged to a single period where only low usage traffic is present [12].

The WiFi/WLAN duty cycle in schools has only been investigated in [25] and for various applications in [24]. No attempt to assess the 24 hour variation of the WiFi duty cycle was made. The duty-cycles in [24] and [25] are similar: median values varied from 1.2 to $1.9 \%$ (standard deviations from 3.1 to $7.1 \%$ or $0.6 \mathrm{~dB}$ ) in [24] and from $1.0 \%$ to $11.7 \%$ or $1.1 \mathrm{~dB}$ with a mean of 4.79\% in [25]. Here, we obtained standard deviations of $1.5 \mathrm{~dB}$, when considering all data 
(Table 3), which is similar. On average, maximal variations of $11.9 \mathrm{~dB}$ (in some schools even up to $30 \mathrm{~dB}$ ) were obtained here. This value is of course much higher than in [24] and [25] because we monitored the duty cycle over 24 hours and in [24] and [25] spatial measurements at different time instances were performed.

Finally, for LTE, [22], [23] proposed extrapolation methods to estimate maximal possible LTE exposure, but no temporal assessment of LTE was performed yet. Variations of LTE were low (average $\mathrm{DR}_{\mathrm{E}, 99}$ of $2 \mathrm{~dB}$ and standard deviation $\sigma_{\mathrm{E}}$ of $0.6 \mathrm{~dB}$ ) and field levels too (maximally $0.022 \mathrm{~V} / \mathrm{m}$ over 24 hours) as the network is very new and being rolled out.

\section{CONCLUSIONS}

Temporal exposures in schools and homes where children are present are assessed and characterized with accurate narrowband equipment (spectrum analyzers and measurement probes) during 24 hours. All present RF signals are considered, including LTE. All values of these signals satisfied the ICNIRP reference levels all day. The largest maximal variations are obtained for the DECT signals $(10.6 \mathrm{~dB})$ and the WiFi signals $(12.7 \mathrm{~dB})$. Electromagnetic fields of broadcasting and telecommunication systems vary maximally 2.9 and $3.3 \mathrm{~dB}$, respectively. Thus, signals from indoor sources produce the largest variations of electromagnetic fields indoor and are the most critical for spatial assessment and comparison with exposure guidelines. Highest field values are measured during the school day. Therefore, we recommend authorities to perform exposure measurements during school days. At night and in the morning the variations of all signals are the lowest and very similar, indicating that night and morning could be merged to a single "exposure" period where only low usage traffic is present. Also the WiFi duty cycles vary most in schools during the school day, while variations of the duty cycles are the lowest at night and in the morning.

Future research should consist of assessing in-situ temporal variations at much more locations and environments. These temporal measurements should be performed during the next years in 
different countries in various microenvironments e.g., within running European projects. When having a larger data set (mainly more microenvironments), one will be able to make more general conclusions. Also the temporal variations of emerging technologies such as LTE are important to characterize as in a few years these networks will be used much more frequently and temporal behavior will change, depending upon the future usage patterns. For LTE it is thus needed to perform each year temporal measurements at fixed locations to investigate the traffic behavior and consequently the temporal variations. Nowadays, the LTE networks are being rolled out but only a limited number of users are present. Thus, only limited temporal variations due to traffic are present. But devices enabled with LTE are being sold and more and more users will make use of the LTE networks, resulting in new traffic and usage patterns.

\section{REFERENCES}

[1] ICNIRP International Commission on Non-ionizing Radiation Protection, Guidelines for limiting exposure to time-varying electric, magnetic, and electromagnetic fields (up to 300 GHz), Health Phys 74(4) (1998) 494-522.

[2] WHO World Health Organization, WHO Research Agenda for Radiofrequency Fields Available at http://www.who.int/peh-emf/research/agenda/en/index.html Accessed 10 January 2013, Geneva, Switzerland, 2010.

[3] W. Joseph, L. Verloock, F. Goeminne, G. Vermeeren, L. Martens, Assessment of general public exposure to LTE and RF sources present in an urban environment, Bioelectromagnetics 31(7) (2010) 576-579.

[4] B. Sirav, N Seyhan, Radio frequency radiation (RFR) from TV and radio transmitters at a pilot region in Turkey, Radiat Prot Dosimetry 136(2) (2009) 114-117. 
[5] L. Verloock, W. Joseph, G. Vermeeren, L. Martens, Procedure for assessment of general public exposure from WLAN in offices and in wireless sensor network testbed, Health Physics 98(4) (2010) 628-638.

[6] W. Joseph, L. Verloock, F. Goeminne, G. Vermeeren, L. Martens, Assessment of RF exposures from emerging wireless communication technologies in different environments, Health Physics 102(2) (2012) 161-72.

[7] J. Tomitsch, E. Dechant, W. Frank, Survey of Electromagnetic Field Exposure in Bedrooms of Residences in Lower Austria. Bioelectromagnetics 31 (2010) 200-208.

[8] W. Joseph, L. Verloock, E. Tanghe, L. Martens, In-situ measurement procedures for temporal RF electromagnetic field exposure of the general public, Health Phys 96(5) (2009) 529-542.

[9] A. Manassas, A. Boursianis, T. Samaras, J.N. Sahalos, Continuous electromagnetic radiation monitoring in the environment: analysis of the results in Greece, Radiat Prot Dosimetry. published online (2012) doi:10.1093/rpd/ncs02.

[10] W. Joseph, L. Verloock, Influence of mobile phone traffic on general public base station exposure, Health Physics 99(5) (2010) 631-638.

[11] Z. Mahfouz, A. Gati, D. Lautru, M.F. Wong, J. Wiart, V.F. Hanna, Influence of Traffic Variations on Exposure to Wireless Signals in Realistic Environments, Bioelectromagnetics 33 (2012) 288-297 doi: 10.1002/bem.20705.

[12] Z. Mahfouz, L. Verloock, W. Joseph, E. Tanghe, A. Gati, J. Wiart, D. Lautru, V.F. Hanna, L. Martens, Comparison of the Temporal Realistic RF Exposure with Worst-Case Estimation in Two Countries, Radiat Prot Dosimetry (2013) doi:10.1093/rpd/nct155.

[13] S. Miclaus, P. Bechet, M. Gheorghevici, Long-term exposure to mobile communication radiation: an analysis of time-variability of electric field level in GSM900 downlink channels, Radiat Prot Dosimetry. 154(2) (2013) 164-73. doi: 10.1093/rpd/ncs16. 
[14] S. Miclaus, P. Bechet, C. Iftode, The application of a channel-individualized method for assessing long-term, realistic exposure to radiofrequency radiation emitted by mobile communication base station antennas. Measurement 46 (2013) 1355-1362.

[15] G. Vermeeren, I. Markaki, F. Goeminne, T. Samaras, L. Martens, W. Joseph, Spatial and temporal RF electromagnetic field exposure of children and adults in indoor micro environments in Belgium and Greece, Progress in Biophysics \& Molecular Biology (2013), accepted 2013.

[16] CENELEC, European Committee for Electrotechnical Standardization, TC 106x WG1 EN 50492 in situ, Basic standard for the in-situ measurement of electromagnetic field strength related to human exposure in the vicinity of base stations, Brussels, Belgium, 2008.

[17] P. Frei, E. Mohler, G. Neubauer, G. Theis, A. Burgi, J. Frohlich, C. Braun-Fahrlander, J. Bolte, M. Egger, M, Roösli, Temporal and spatial variability of personal exposure to radiofrequency electromagnetic fields, Environmental Research 109(6) (2009) 779-785.

[18] W. Joseph, P. Frei, M. Roösli, G. Thuróczy, P. Gajsek, T. Trcek, J. Bolte, G. Vermeeren, E. Mohler, P, Juhasz, V. Finta, L. Martens, Comparison of personal radio frequency electromagnetic field exposure in different urban areas across Europe, Environmental Research 110 (2010) 658 - 663.

[19] M. Röösli, P. Frei, J. Bolte, G. Neubauer, E. Cardis, M. Feychting, P. Gajsek, S. Heinrich, W. Joseph, S. Mann, L. Martens, E. Mohler, R. Parslow, A.H. Poulsen, K. Radon, J. Schüz, G. Thuroczy, J.-F. Viel, M. Vrijheid, Proposal of a study protocol for the conduct of a personal radiofrequency electromagnetic field measurement campaign. Environmental Health (2010) $9-23$.

[20] J. Bolte, G. Van der Zande, J. Kamer, Calibration and Uncertainties in Personal Exposure Measurements of Radiofrequency Electromagnetic Fields, Bioelectromagnetics 32(8) (2011) 652-663 DOI 10.1002/bem.20677. 
[21] W. Joseph, L. Verloock, L. Martens, Reconstruction of the Polarization Ellipse of the EM field of Base Station Antennas by a Fast and Low-cost Measurement Method, IEEE Trans. Electromag. Compat. 48(2) (2006). 385 - 396

[22] L. Verloock, W. Joseph, A. Gati, N. Varsier, J. Wiart, L. Martens, B. Hansson, low-cost extrapolation method for maximal LTE radio base station exposure estimation: test and validation, Radiat Prot Dosimetry (2012) 1-5, doi:10.1093/rpd/ncs307

[23] W. Joseph, L. Verloock, F. Goeminne, G. Vermeeren, and L. Martens, In-situ LTE exposure of general public: characterization and extrapolation, Bioelectromagnetics 33(6) (2012) 466-475.

[24] W. Joseph, D. Pareit, G. Vermeeren, D. Naudts, L. Verloock, L. Martens, I. Moerman, Determination of the duty cycle of WLAN for realistic radio frequency electromagntic field exposure assessment, Progress in Biophysics \& Molecular Biology 111 (2013) 30-36, 2013.

[25] Khalid, M., Mee, T., Peyman, A., Addison, D., Calderon, C., Maslanyj, M., Man, S., Exposure to radio frequency electromagnetic fields from wireless computer networks: Duty factors of Wi-Fi devices operating in schools, Progress in Biophysics and Molecular Biology 107(3) (2011) 412-420. 
Acknowledgement: W. Joseph is a Post-Doctoral Fellow of the FWO-V (Research FoundationFlanders). The study was commissioned, financed, and steered by the Flemish government, Department of Environment, Nature and Energy (LNE).

The Flemish government, Department of Environment, Nature and Energy (LNE) commissioned this study and no separate ethical approval was needed as the measurements did not involve the children self: the measurements were performed in microenvironments where children can be or were present. Approval from schools and Flemish government was obtained. 


\section{List of captions}

Table 1: Maximal measured electric field $E_{\max }$, exposure ratio $E R$, and average values of $D R_{E, \max }$, $D R_{E, 99}$, and the standard deviation $\sigma_{E}$ of the momentary measured field values $E_{\text {mom }}$ for different signal types for the considered microenvironments schools and homes.

Table 2: Influence of different time instances (day morning, school day, evening, night) on the average values of $D R_{E, \max }, D R_{E, 99}$, and the standard deviations.

Table 3: Average values of $D R_{D C \text {,max }}, D R_{D C, 99}$, and the standard deviation $\sigma_{D}$ of the WiFi duty cycle per microenvironment and for different time instances of the day.

Figure 1: Variation of the momentary electric field strength produced by different external RF sources measured with a spectrum analyzer during 24 hours in schools and homes; (a) FM, (b) TDAB, (c) DVB-T, (d) TETRA, (e) GSM900, (f)GSM1800, (g) UMTS/HSPA, (h) LTE.

Figure 2: Variation of the momentary electric field strength produced by different internal RF sources measured with a spectrum analyzer during 24 hours in schools and homes; (a) DECT, (b) WiFi 2.4 GHz.

Figure 3: Variation of the momentary duty cycle of the WiFi $2.4 \mathrm{GHz}$ signal transmitted in channel 11, measured with the spectrum analyzer during 24 hours in a school. 


\begin{tabular}{llccccc}
\hline environment & RF source & $\begin{array}{c}\mathrm{E}_{\max } \\
(\mathrm{V} / \mathrm{m})\end{array}$ & $\begin{array}{c}\mathrm{ER} \\
(\%)\end{array}$ & $\begin{array}{c}\mathrm{DR}_{\mathrm{E}, \max } \\
(\mathrm{dB})\end{array}$ & $\begin{array}{c}\mathrm{DR}_{\mathrm{E}, 99} \\
(\mathrm{~dB})\end{array}$ & $\begin{array}{c}\sigma_{\mathrm{E}} \\
(\mathrm{dB})\end{array}$ \\
\hline schools & broadcasting & 0.16 & 0.56 & 2.4 & 2.0 & 0.4 \\
& telecommunication & 1.13 & 2.74 & 3.8 & 2.9 & 0.7 \\
& DECT & 3.10 & 5.20 & 13.4 & 11.8 & 2.9 \\
& WiFi 2.4 GHz* & 1.70 & 2.78 & 12.6 & 7.7 & 1.6 \\
\hline homes & broadcasting & 0.23 & 0.84 & 3.2 & 2.5 & 0.5 \\
& telecommunication & 0.12 & 0.28 & 3.0 & 2.3 & 0.5 \\
& DECT & 0.16 & 0.27 & 8.5 & 6.3 & 1.4 \\
& WiFi 2.4 GHz* & 0.68 & 1.12 & 12.7 & 9.1 & 1.8 \\
\hline all & broadcasting & 0.23 & 0.84 & 2.9 & 2.3 & 0.5 \\
& telecommunication & 1.13 & 2.74 & 3.3 & 2.6 & 0.6 \\
& DECT & 3.10 & 5.20 & 10.6 & 8.7 & 2.1 \\
& WiFi 2.4 GHz* & 1.70 & 2.78 & 12.7 & 8.5 & 1.7 \\
\hline
\end{tabular}

*only 1 vector component of the electric field is measured for the temporal variation $\mathrm{s}$ of this signal.

FM: Frequency Modulation, PMR: Private Mobile Radio, T-DAB: Terrestrial-Digital Audio Broadcasting, TETRA: Terrestrial Trunked Radio, DVB-T: Digital Video Broadcasting-Terrestrial, GSM: Global System for Mobile Communications, LTE: Long Term Evolution, DECT: Digital Enhanced Cordless Telecommunications, UMTS: Universal Mobile Telecommunications System, HSPA: High Speed Packet Access, WiFi: Wireless Fidelity 802.11 (WiFi at $2.4 \mathrm{GHz}$ and WiFi at $5 \mathrm{GHz}$ ).

Telecommunication includes here GSM900, GSM1800, UMTS/HSPA, TETRA, and LTE. Broadcasting signals include FM, T-DAB, DVB-T.

Table 1 


\begin{tabular}{llccc}
\hline time instance & RF source & $\begin{array}{c}\mathrm{DR}_{\mathrm{E}, \max } \\
(\mathrm{dB})\end{array}$ & $\begin{array}{c}\mathrm{DR}_{\mathrm{E}, 99} \\
(\mathrm{~dB})\end{array}$ & $\begin{array}{c}\sigma_{\mathrm{E}} \\
(\mathrm{dB})\end{array}$ \\
\hline morning & DECT & 6.3 & 5.1 & 1.0 \\
& WiFi $2.4 \mathrm{GHz}$ & 5.1 & 3.9 & 0.7 \\
& cumulative external sources & 2.1 & 1.8 & 0.3 \\
\hline school day & DECT & 10.2 & 8.9 & 2.5 \\
& WiFi 2.4 GHz & 8.9 & 6.3 & 1.3 \\
& cumulative external sources & 3.0 & 2.6 & 0.6 \\
\hline evening & DECT & 7.9 & 5.2 & 1.4 \\
& WiFi 2.4 GHz & 9.9 & 7.7 & 1.7 \\
& cumulative external sources & 2.6 & 2.2 & 0.4 \\
\hline night & DECT & 6.4 & 4.9 & 1.0 \\
& WiFi 2.4 GHz & 5.3 & 3.6 & 0.6 \\
& cumulative external sources & 2.1 & 1.8 & 0.3 \\
\hline
\end{tabular}

Table 2 


\begin{tabular}{llccc}
\hline & & $\begin{array}{c}\mathrm{DR}_{\mathrm{DC}, \max } \\
(\mathrm{dB})\end{array}$ & $\begin{array}{c}\mathrm{DR}_{\mathrm{DC}, 99} \\
(\mathrm{~dB})\end{array}$ & $\begin{array}{c}\sigma_{\mathrm{DC}} \\
(\mathrm{dB})\end{array}$ \\
\hline environment & schools & 30.6 & 10.9 & 2.9 \\
& homes & 6.4 & 4.3 & 0.9 \\
& all data & 11.9 & 6.3 & 1.5 \\
\hline time instance & morning & 2.0 & 1.9 & 0.2 \\
& school day & 12.7 & 9.8 & 1.9 \\
& evening & 4.9 & 4.3 & 1.1 \\
& night & 1.9 & 1.8 & 0.2 \\
\hline
\end{tabular}

Table 3 


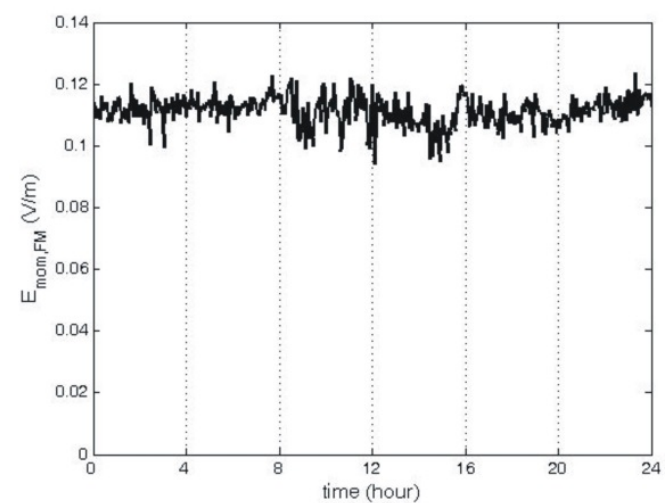

(a)

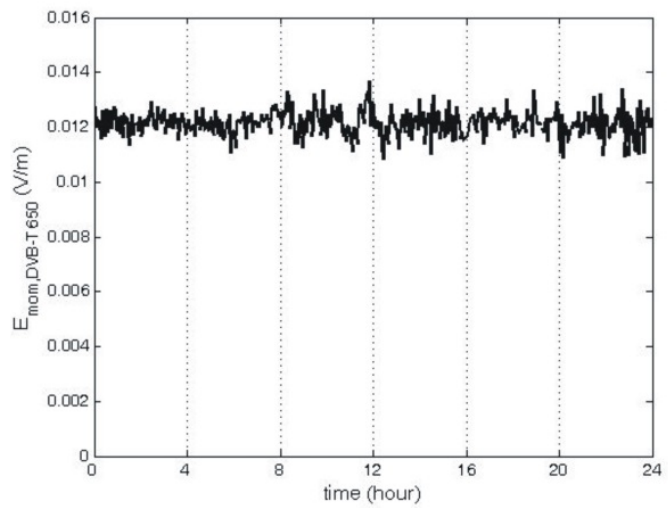

(c)

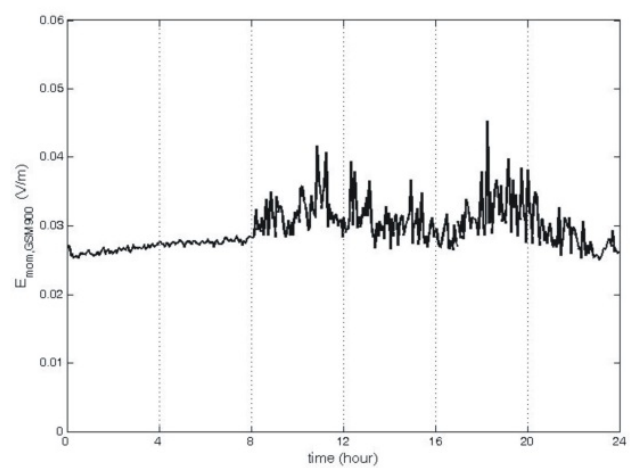

(e)

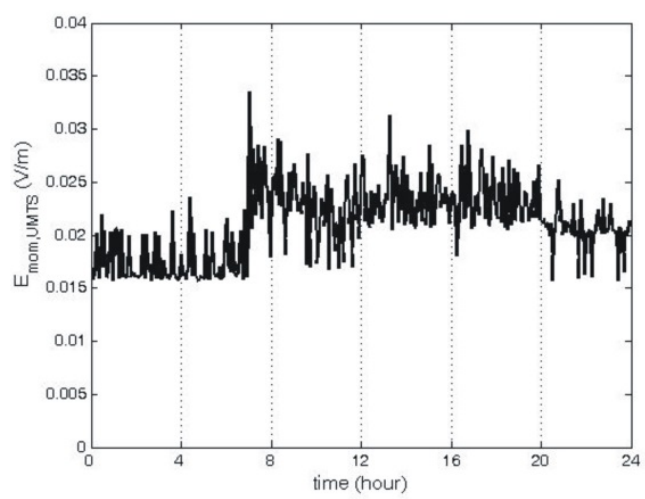

(g)

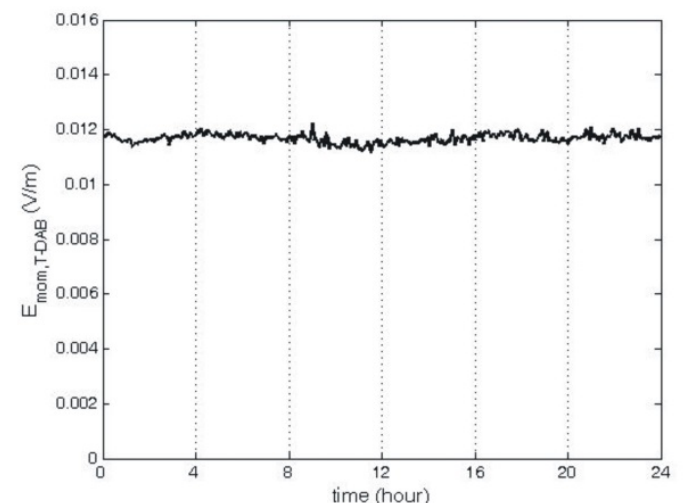

(b)

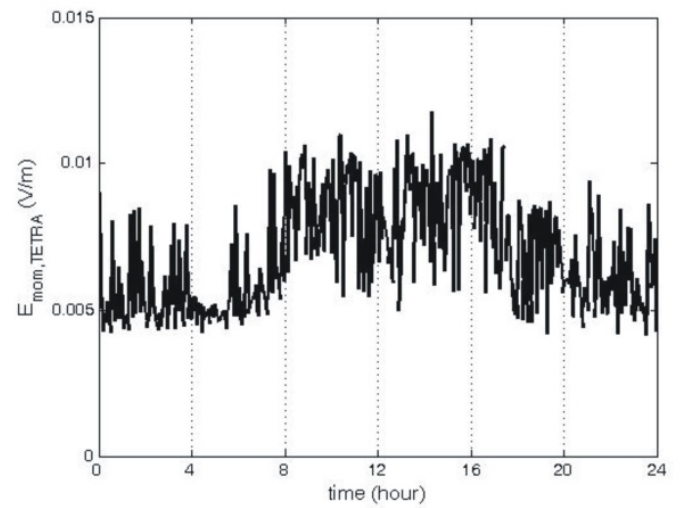

(d)

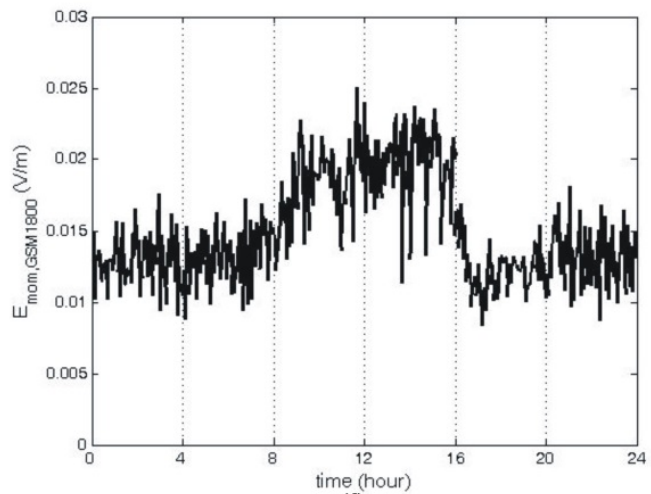

(f)

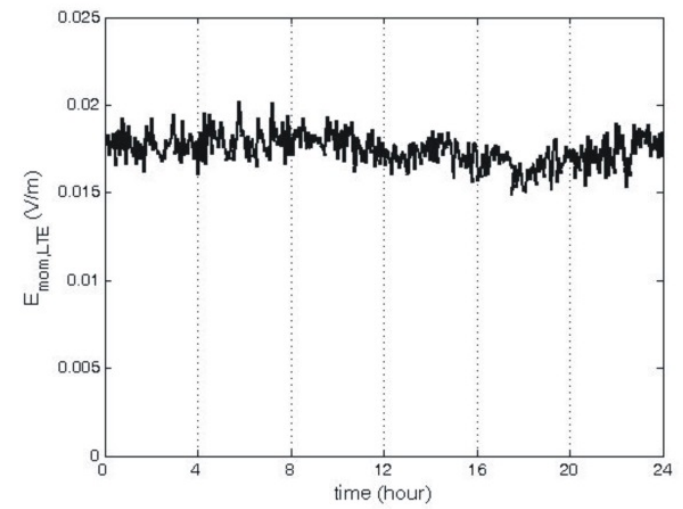

(h)

Figure 1 


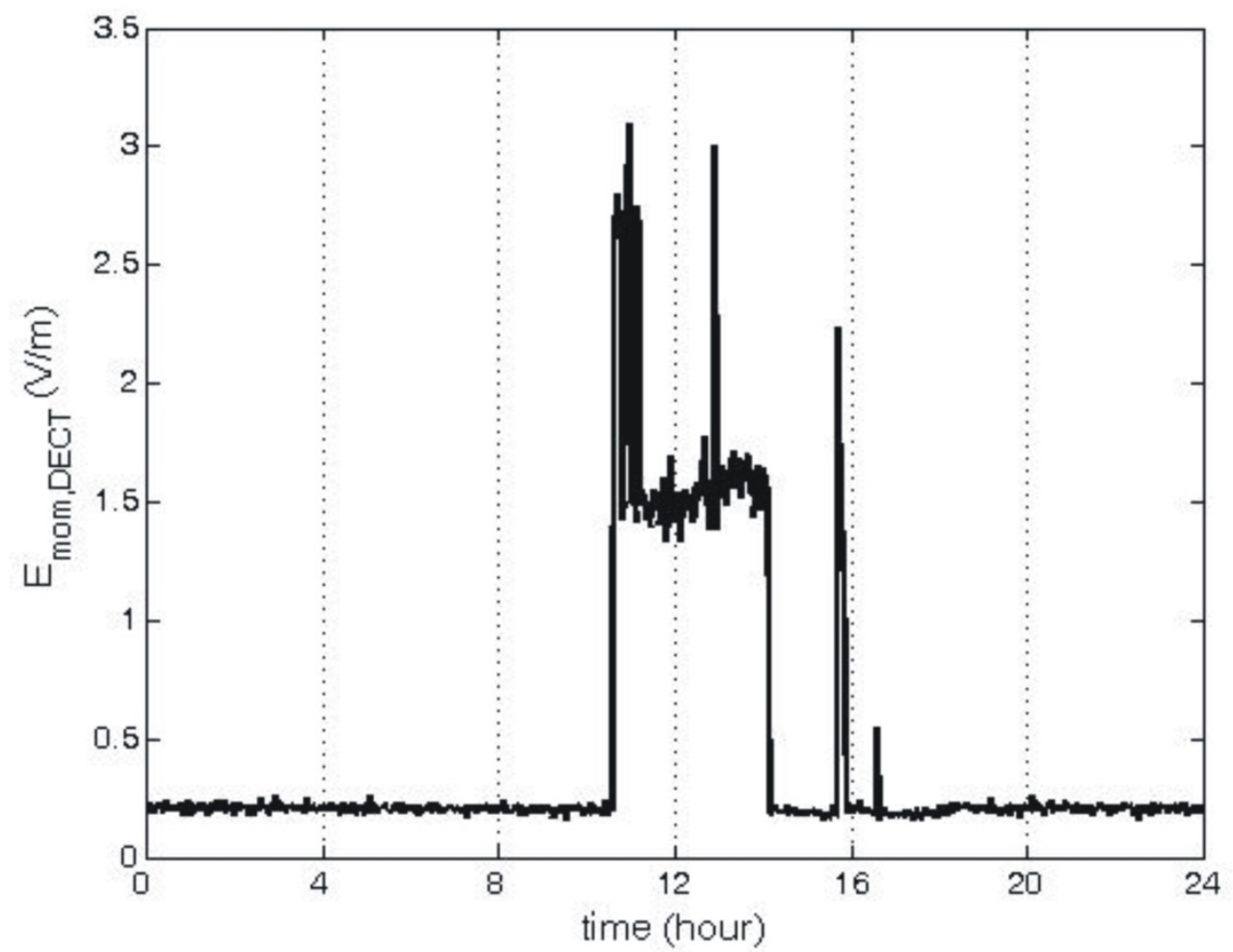

(a)

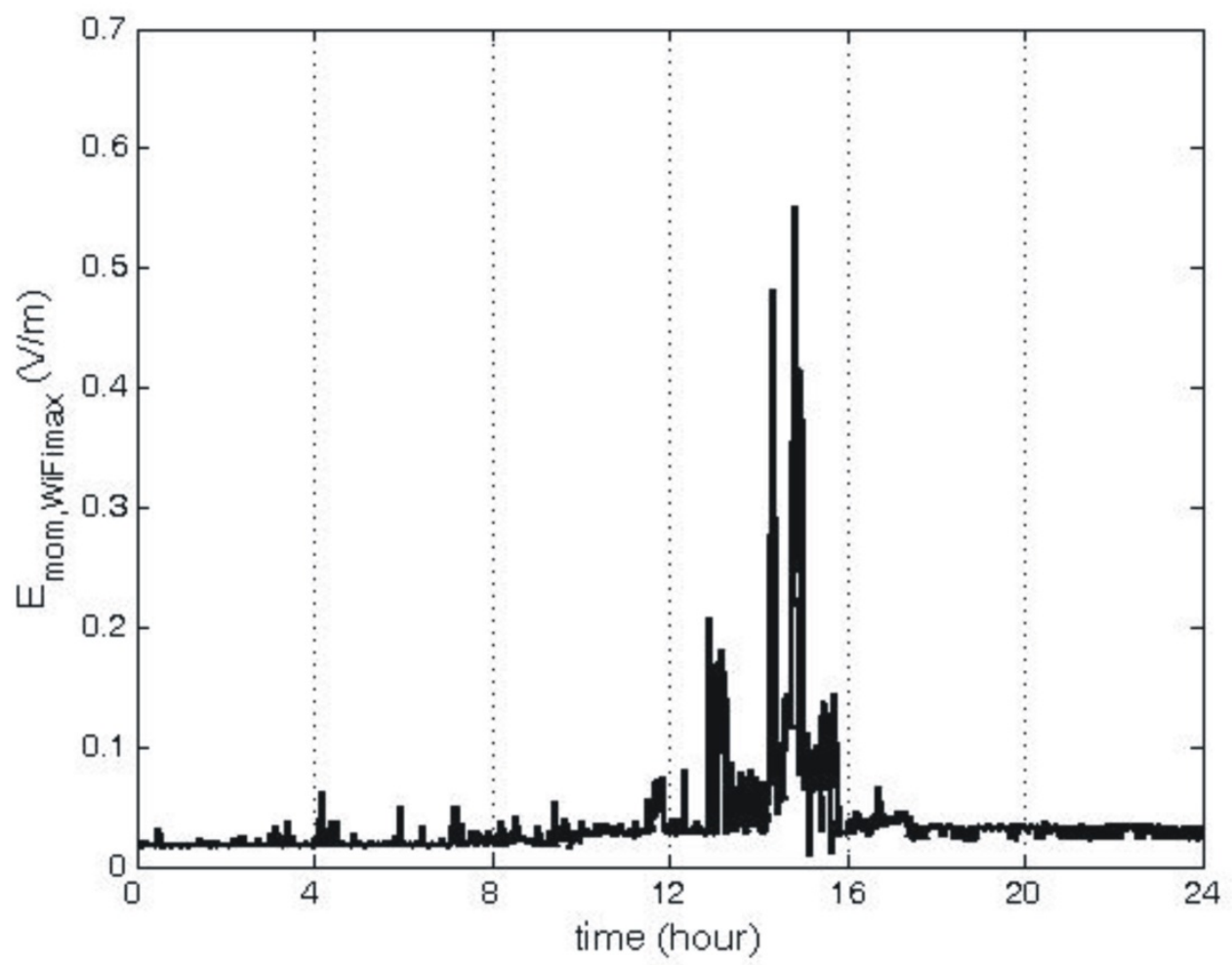

(b)

Figure 2 


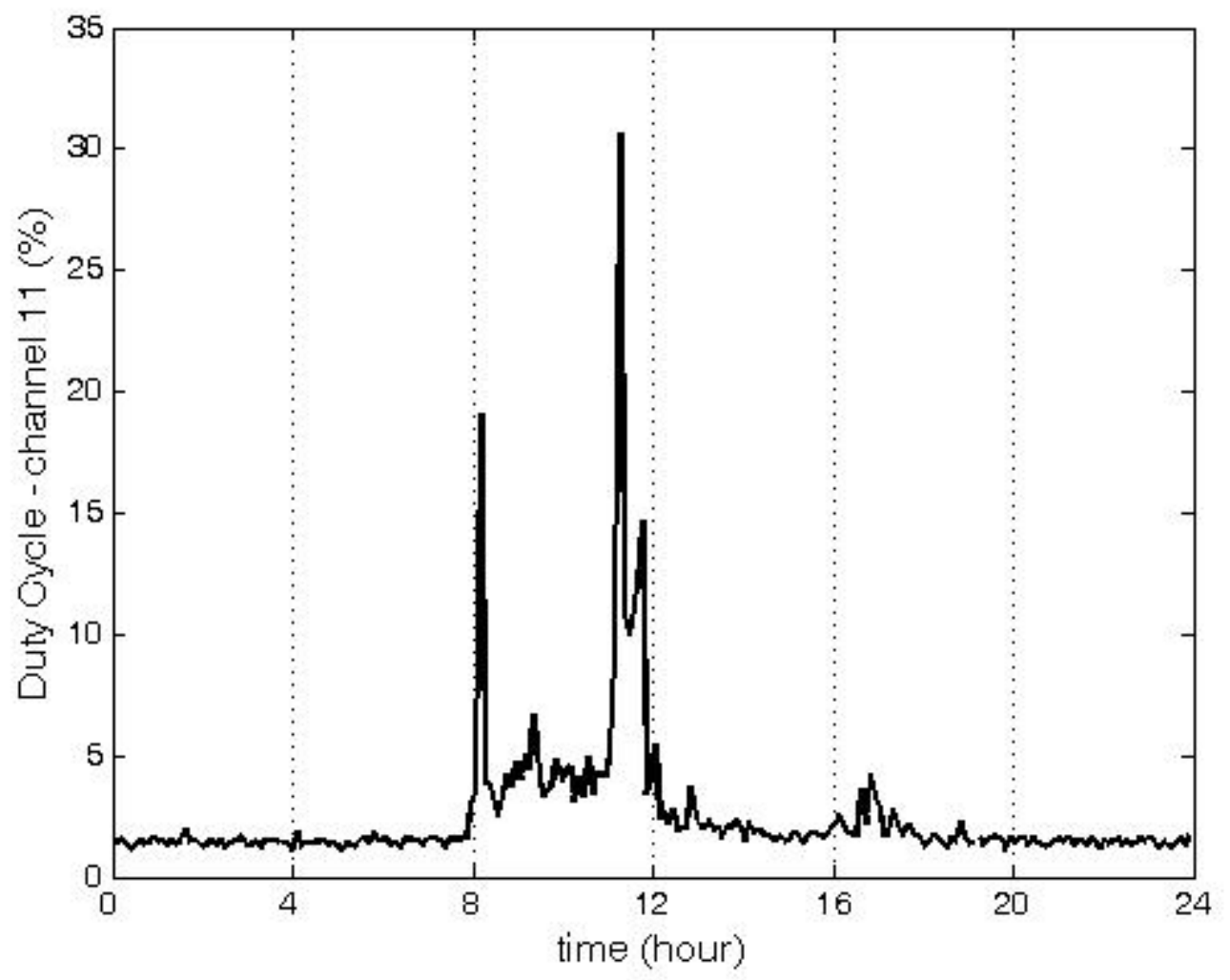

Figure 3 